\title{
Implicitly activated memories are associated to general context cues
}

\author{
Douglas L. Nelson, Leilani B. Goodmon, ANd Umit AKIRMAK \\ University of South Florida, Tampa, Florida
}

\begin{abstract}
Words having more densely entangled associative structures are more likely to be recalled in the presence of related extralist cues. A context-modified PIER2 model predicts that the implicit activation of such structures during study connects them to the context of the learning episode. In two experiments, we evaluated this assumption by varying the associative density of the study words and the accessibility of context information. In Experiment 1, we varied context accessibility by manipulating context awareness and by delaying testing and filling the delay with different types of interfering tasks. In Experiment 2, we varied accessibility by manipulating test delay and type of interference in a factorial design. The effects of associative density were reduced by using implicit testing that never refers to context, by performing interpolated interference tasks, and finally, by extending the length of the delay. Information that is implicitly activated during an episodic experience is associated to the context of that experience.
\end{abstract}

Words become associated with each other through contiguous experience and exist as locally and globally entangled representations within the associative space that binds the lexicon together (Nelson, McKinney, Gee, \& Janczura, 1998). Local associative structures for individual words differ in how many associates they have and how connected these associates are with each other and the word itself. Given the use of free association probability as the metric for assessing word-to-word strength, words differ in network size and connectivity. The existence of such structures becomes readily apparent whenever words are encountered in isolation. Seeing or hearing a contextually isolated word activates related words and the links that bind them together; for example, seeing food activates food-eat, food-drink, food-hunger, eat-hunger, eat-food, and so on. When an encoding context is absent or ambiguous, the lexical system provides context by activating the word's associative history, and the complexity of this history affects its recall and recognition.

Research findings indicate that studied target words with higher levels of associative connectivity are more likely to be recognized (Nelson, Zhang, \& McKinney, 2001), recalled in primed free association (Nelson \& Goodmon, 2003), and recalled in extralist cuing (e.g., Nelson, Bennett, Gee, Schreiber, \& McKinney, 1993). Memory is affected by remote associate-to-associate links (eat-hunger), and the effects of such links are not contingent on links back to the target (Nelson, McEvoy, \& Pointer, 2003). Extralist cuing research shows that the effects of connectivity are similar for words varying in printed frequency, concreteness, and network size (Gee, Nelson, \& Krawczyk, 1999; Nelson, Bennett, et al., 1993;
Nelson \& Goodmon, 2003). Higher levels of connectivity facilitate recall in extralist cuing for young and old participants, after short or long study times, after one or two study trials, and under incidental and intentional learning and testing conditions (Nelson, Bennett, et al., 1993; Nelson \& Goodmon, 2002, 2003). In addition, after adjusting for other differences, lexical decision and naming times are slower for words having higher levels of connectivity (Balota, Cortese, Sergent-Marshall, Spieler, \& Yap, 2004).

Associative connectivity has robust effects, and the present experiments investigate the sensitivity of these effects to disruptions of general context cues. General context cues refer to the physical, social, and emotional cues linked to the environment in which words are studied. Existing research indicates that context disruptions reduce episodic recall (e.g., Mensink \& Raaijmakers, 1988; Smith \& Vela, 2001), and the issue is whether such disruptions also reduce associative connectivity effects. Such effects are produced by the implicit activation of long-term memories (Nelson et al., 1998), and context disruptions may have no effect on the retrieval of such memories. The activation occurs in the novel context provided by the experimental session, but implicitly activated information may not be directly associated to the context in which the activation takes place. In the extralist cuing task, associative connectivity effects linked to the target may be determined solely by an extralist cue that reinstates the activation during testing. Alternatively, the memory system may connect both what is explicitly encoded and what has been implicitly activated to the general context provided by the environment.

D. L.Nelson, dnelson@cas.usf.edu 
To date, only two studies on the relationship between general context cues and the effects of associative connectivity have been reported. One shows that connectivity effects are reduced in primed free association in comparison with cued recall (Nelson \& Goodmon, 2003), and the other shows that, in cued recall, connectivity effects are reduced after $10 \mathrm{~min}$ of solving multiplication problems (Nelson et al., 1998). These results suggest that connectivity effects become associated to general context cues during learning. The free association-cued recall contrast manipulates context awareness. In primed free association, the targets are processed incidentally during study and the test instructions do not refer to the relation between the test cues and the words appearing in the study episode. Context is less likely to be recovered during retrieval because context is less likely to be encoded under incidental learning conditions and because the test instructions discourage the use of context as a cue during testing. The math task reduces the power of context cues available during retrieval to recover context information encoded during study, because it introduces a test delay. It also creates a second processing context that may compete with the recovery of the list-learning context.

The present experiments subject target connectivity effects to a more extensive range of context disruptions. Experiment 1 varies target connectivity, target frequency, interference condition, and context awareness. We varied interference by testing immediately after the test instructions, after 8 min of easy or difficult multiplication, or after studying a second list of words. The second word list was either unrelated or related to the test cues. If target connectivity effects are linked to context, then such effects will diminish after problem solving and list-learning tasks, in comparison with the immediate test. We varied context awareness by comparing target recovery under primed free association and extralist cuing conditions. If connectivity effects are linked to context, such effects will be smaller in primed free association than in cued recall. In cued recall, participants know that memory for the study words will be tested and they know that each test cue is associatively related to one of the words appearing in the study list. In primed free association, the target words are processed incidentally, and the test instructions never mention the study context. They encourage participants to produce the first word that comes to mind that is related to the test cue. If implicitly activated associates of the target are connected to context during study, the effects of target connectivity will interact with both interference and context awareness. Finally, we varied printed frequency to determine whether target connectivity and frequency effects respond similarly to context disruptions. Normally, low-frequency words are more likely to be recalled in extralist cuing (e.g., Nelson \& McEvoy, 2000; Nelson \& Zhang, 2000), but the mechanism underlying this result is unclear. Results showing that both variables respond similarly to the interference and awareness manipulations would support the interpretation that both connectivity and frequency represent implicit activation effects (Nelson et al., 1998).

In Experiment 1, retention interval covaries with the difference between problem solving and list-learning in- terference. We intentionally confounded interval, because presenting the interference list required only $1.2 \mathrm{~min}$, an interval deemed too short to produce interference in the multiplication task. Previous findings have indicated that target recall is higher after the math task than after list learning (Nelson, McEvoy, Janczura, \& Xu, 1993), and the interval-task confounding represented an attempt to equate recall in the two interference conditions. Experiment 2 eliminates the confounding by using short delays commensurate with those used in processing the interference list. Extralist cued recall on an immediate test is compared with recall after short delays filled by problem solving or additional list learning. Given short retention intervals and the assumption that context mismatches are more likely after list learning, Experiment 2 tests the hypothesis that list learning produces greater reductions in target connectivity effects than does problem solving.

\section{EXPERIMENT 1}

A context-modified version of PIER2 (processing implicit and explicit representations; Nelson, Goodmon, \& Ceo, 2007) guided expectations for this experiment. In PIER2, the associative structure of the target is measured by using free association to determine what its associates are and how strongly they are connected with each other and with the target (structures for 4,000 words are available in Nelson, McEvoy, \& Schreiber, 2004). Figure 1 illustrates the three types of links that make up the target's structure: target to associate, associate to associate, and associate to target. Two targets with five associates are shown. The associates of these words have been assigned equivalent target-to-associate strengths, represented as arrows from the target's implicit representation $t$ to each associate. Word $\mathrm{T}_{1}$ has higher activation strength than $\mathrm{T}_{2}$, because it has more associate-to-associate and associateto-target links. Associate-to-associate links connect the target's associates, and associate-to-target links connect these associates to the target. The target's inactivated state evolves to an activated state when the target is seen or heard, and its activated state is computed by adding target $t$ self-strength (1.0) to the sum of the strengths of the three kinds of links (Nelson et al., 2003). The computation ignores link origin and termination, and target activation increases directly with the summed strength of the links that make up its network. In short, target activation strength equals the sum of the associative history of its interactions with other words appearing in various contexts.

To clarify the relationship between activation strength and context, we incorporated the interactive cue assumption of SAM (search of associative memory; Raaijmakers \& Shiffrin, 1981; Mensink \& Raaijmakers, 1988; see also Humphreys, Tehan, O'Shea, \& Bolland, 2000) into a context-modified version of PIER2. In this version, the activated representation of the target and its strengthening as a result of explicit encoding operations become associated to context during study. During retrieval, extralist and context cues jointly determine the probability of the target's recovery (see Nelson et al., 2007, for details and a computational example). Target recovery is a probabilistic function 

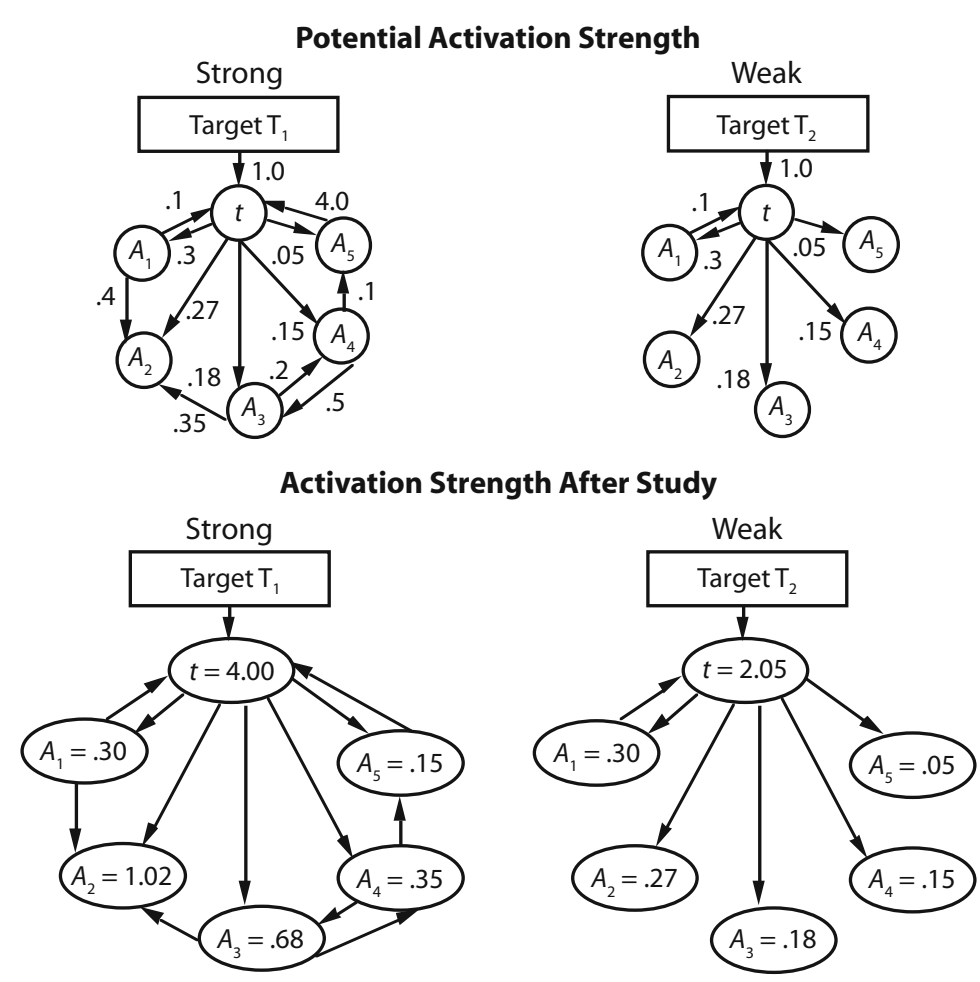

Figure 1. Potential and realized target activation strength after study, according to PIER2.

of the target's implicit and explicit encodings adjusted by retention interval and a context parameter that varies with the disruption task (e.g., context awareness, multiplication, list learning). ${ }^{1}$ This adjustment reduces the influence of the target's encodings by multiplying predicted strength by test delay raised to a negative power of the context parameter. This constraint causes the model to predict that the effects of target activation strength will decline as a power function of the length of the retention interval, with the rate of decline determined by the disruption task. Additional tasks performed after study add contexts and increase the probability of context mismatches during testing. They reduce the probability of sampling the context associated with the first task (Mensink \& Raaijmakers, 1988).

The context-modified model predicts that each interference condition will reduce the recall of the initial list. Additionally, differences between interference conditions can emerge. Context cues are likely to be more similar for two word lists than for a word list and a multiplication task. If they are, context mismatches will be more likely for listlearning interference than for problem solving interference when retention interval is equated. However, by differentially lengthening the retention interval for problem solving, this task can theoretically be made to generate as much interference as list learning. In the model, interference is determined by both test delay and the likelihood of context mismatching. Furthermore, interference manipulations that fail to increase context mismatching should fail to influence effects of target activation strength. For example, because the contexts are likely to be similar for easy and hard math tasks, difficulty should not influence context mismatching and should have no effect. In contrast, if both extralist and context cues jointly control target recovery, the relatedness of words appearing in the second study list to the test cues should affect context mismatching. When the words of the interference list are unrelated to the test cues, context mismatching is likely to occur, but the amount of mismatching should be less than that for related lists. The words studied in the related list are strongly associated to the test cues. In the related condition, the extralist cues are likely to recover words from both contexts, which should increase the probability of context mismatches beyond the baseline defined by unrelated lists.

\section{Method}

Design and Participants. We used a $5 \times 2 \times 2 \times 2$ mixed-model design, with context interference, context awareness, and target frequency varied between participants, and target activation strength varied within participants. Targets were frequently or infrequently occurring words, and target activation strength was strong or weak. Testing immediately followed the test instructions or was delayed by easy or difficult multiplication tests or by studying a second word list that was either unrelated or related to the test cues used to prompt recall. Context awareness was varied by contrasting extralist cued recall with primed free association. Sixteen participants served in each between-subjects condition, and 320 served in the experiment, assigned in randomized replication blocks to conditions and lists. In exchange for their service, participants met a psychology course requirement and received course credit.

Materials. Two 24-item study lists were used for each frequency level as well as for the context interference lists (see the Appendix). 
Table 1

Mean Strengths and Standard Deviations for Targets $T_{1}$ and $T_{2}$

\begin{tabular}{|c|c|c|c|c|c|}
\hline \multirow[b]{2}{*}{ Manipulated Variable } & \multirow[b]{2}{*}{ Definition } & \multicolumn{2}{|c|}{$\mathrm{T}_{1}$} & \multicolumn{2}{|c|}{$\mathrm{T}_{2}$} \\
\hline & & $M$ & $S D$ & $M$ & $S D$ \\
\hline Target Frequency & Printed frequency per million words & 5 & 4 & 308 & 321 \\
\hline Target Activation Strength & Sum of link strengths comprising the target's network & 3.06 & 0.80 & 6.18 & 1.51 \\
\hline Associate-to-associate strength & Sum of link strengths between the associates of the target & 0.66 & 0.43 & 3.51 & 1.24 \\
\hline Associate-to-target strength & Sum of link strengths from the associates to the target & 0.57 & 0.63 & 0.81 & 0.81 \\
\hline Target self-strength & Target self-strength & 1.00 & & 1.00 & \\
\hline Target-to-associate strength & Sum of link strengths from the target to its associates & 0.83 & 0.07 & 0.86 & 0.06 \\
\hline
\end{tabular}

Table 1 presents item characteristics and definitions for manipulated variables. As can be seen, low- and high-frequency targets occurred approximately 5 and 308 times per million words, respectively. All words were selected so that all participants would be likely to understand them; that is, the nonresponse rate in free association is low at each level of frequency. Target activation strength indexed by the summation rule averaged 6.18 when high and 3.06 when low. Targets with higher and lower activation levels had averages of 2.98 ( $S D=$ $.76)$ and $.77(S D=.43)$ associate-to-associate links, respectively, and averages of $1.81(S D=.65)$ and $1.74(S D=.58)$ associate-totarget links. Target activation was equated at each level of frequency in terms of mean strength and in terms of the results of a simultaneous regression analysis on the lists with target activation strength as the dependent measure. Printed frequency and the link strengths that comprised target activation strength were used as predictors. The results of the analysis indicated that frequency and target activation strength were uncorrelated (partial $r=-.09$ ), and that both associate-to-associate and associate-to-target links were significant predictors of target activation strength. A subsequent stepwise regression indicated that associate-to-associate strength explained more variance $(86.5 \%)$ than did associate-to-target strength $(12.2 \%)$, so links between the associates provided the primary source of variation in target activation strength.

Other variables known to affect cued recall were controlled (see Table 2). Forward and backward strengths were set at weak-moderate levels of strength, as were associate-to-cue, shared associate, and mediated strengths. These variables were set to avoid floor and ceiling effects. Similarly, variables that decrease recall were controlled at moderate levels. Each cue produced an average of 7.89 ( $S D=$ 4.16) competitors at an average strength of .36, and each target generated an average of $8.55(S D=4.51)$ competitors at an average strength of .46. Competitor strength for a given cue-target pairing was determined by summing the strengths of each competitor.

For the interference lists, words in the unrelated lists had no direct links to List 1 target words or to their cues. Words in the related lists, on average, had only weak forward (.06) and backward (.03) links to List 1 target words, but the strengths of these links were variable, with the majority at zero. In contrast, the test cues were strongly associated to both the List 1 targets and to the List 2 words of the related interference list. To provide benchmark comparisons for how strongly the test cues were associated to both lists, PIER's predicted recall probability for extralist cued recall on immediate tests was computed for each list. The model predicted probabilities of .52 and .51 , respectively, for target list recall and related interference list recall. The words from the related list had a high potential for producing strong competition for the words of the target list.

Procedure. Data were collected in individual sessions in which participants were asked to read all study words aloud when they were shown. Each word was presented for $3 \mathrm{sec}$ on a computer monitor, and prior to the study trial, a short list of proper names was shown to familiarize participants with the rate. Cued recall participants were asked to remember as many words as possible but were not told how they would be tested. Primed free association participants were asked to rate each study word for concreteness on a 1-5 scale. Concreteness was explained, the scale was printed on a card that was always available, and high ratings indicated a higher concreteness rating. The cover story indicated that we would be asking participants to perform several different tasks that would allow normative data collection. Testing memory was never mentioned. The same study instructions were used with the interference lists when the design called for a second list.

In the immediate test conditions, participants were given the test trial instructions following the last study word. The cued recall instructions indicated that cue words would be shown one at a time and that each cue was meaningfully related to one studied word. Participants were told to read the cue aloud and then recall its target; they were told that they could guess. The primed free association instructions asked participants to produce the first associatively related word to come to mind with each test cue. The study episode was never mentioned, and an additional 24 cues were added to the test trial to reduce the likelihood of participants' discovering the relation between the cues and the study words. The distractors were ran-

Table 2

Mean Strengths and Standard Deviations for Cue-Target Links

\begin{tabular}{|c|c|c|c|}
\hline \multirow[b]{2}{*}{ Controlled Variable } & \multirow[b]{2}{*}{ Definition } & \multicolumn{2}{|c|}{ Cue Recall } \\
\hline & & M & $S D$ \\
\hline Cue-Target Links & Links that join cue and target & & \\
\hline Forward strength & Cue-to-target link & .12 & .05 \\
\hline Backward strength & Target-to-cue links & .08 & .11 \\
\hline Associate-to-cue strength & Links from the target's associates to the test cue & .20 & .27 \\
\hline Shared associate strength & An associate produced by cue and target & .05 & .08 \\
\hline Mediated strength & Cue-to-mediating associate-to-target links & .03 & .06 \\
\hline Competitors & Associates unique to cue or to target & & \\
\hline Cue competitor strength & Associates unique to cue & .36 & .24 \\
\hline Target competitor strength & Associates unique to target & .46 & .25 \\
\hline Target accessibility & Number of words that produce the target as an associate (out of 5,000 ) & 31.55 & 41.64 \\
\hline Target concreteness & Rated concreteness on a $1-7$ scale & 4.80 & 1.29 \\
\hline
\end{tabular}


domly intermixed with the extralist cues during testing and were not directly associated with any of the targets or the test cues. Adding distractors to the extralist cuing task does not affect recall, and they were omitted in this task (Nelson \& Goodmon, 2003). The test trial for all participants was self-paced, and the orders of all presented words were randomized independently for each participant.

Participants in the delayed testing conditions were given the same test instructions after they completed the intervening task. After the last study word, participants receiving delayed testing were told about their intervening task. In the math conditions, the problems were shown on the screen for a given period, followed by the appearance of a question mark for $2 \mathrm{sec}$, at which time participants were to report the solution. In the easy math condition (e.g., $8 \times 4$ ), 96 problems were shown for $3 \mathrm{sec}$ each, and in the hard math condition (e.g., $57 \times 6$ ), 48 problems were shown for $8 \mathrm{sec}$ each. This procedure set the retention interval length to $8 \mathrm{~min}$ in each condition. In list learning, participants were told that a second list of words would be presented in the same manner as the first list had been, and that they should read the words aloud and attempt to remember as many as possible. The words in the interfering lists were shown at a 3-sec rate, which produced a shorter retention interval of $1.2 \mathrm{~min}$, in comparison with the 8-min interval used for the math tasks. The longer retention interval for math was used to equate target recall so that target activation effects for different tasks could be compared without confounding the comparison with level of recall. Finally, the sequences of the studied words appearing on the second list were randomized for each participant independently of the first list. After the last interfering word was shown, either cued recall or primed free association instructions were provided.

\section{Results and Discussion}

Figures 2 and 3 present the probabilities of target recovery (and $S E$ s) for extralist cued recall and primed free association, respectively. In each figure, target recovery is displayed as a function of interference condition, target activation, and target frequency. The experiment generated a large amount of data, but the findings were orderly and straightforward. Figure 2 shows that recall increased when testing was immediate rather than delayed by the interference manipulations. Recall also increased when target frequency was lower as opposed to higher, and when target activation was stronger as opposed to weaker. A similar
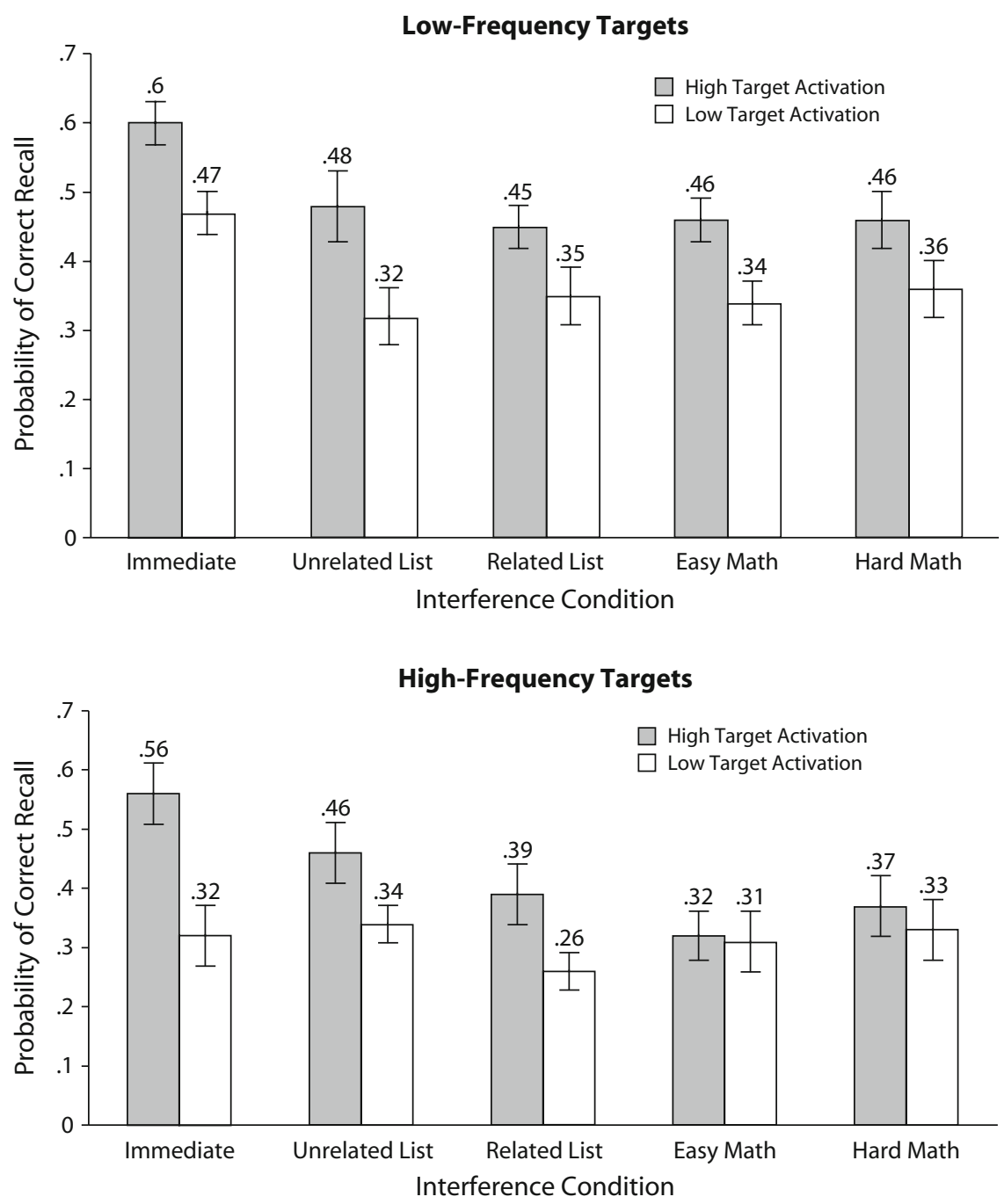

Figure 2. Probability of extralist cued recall in Experiment 1, as a function of context interference, target frequency, and target activation strength. 

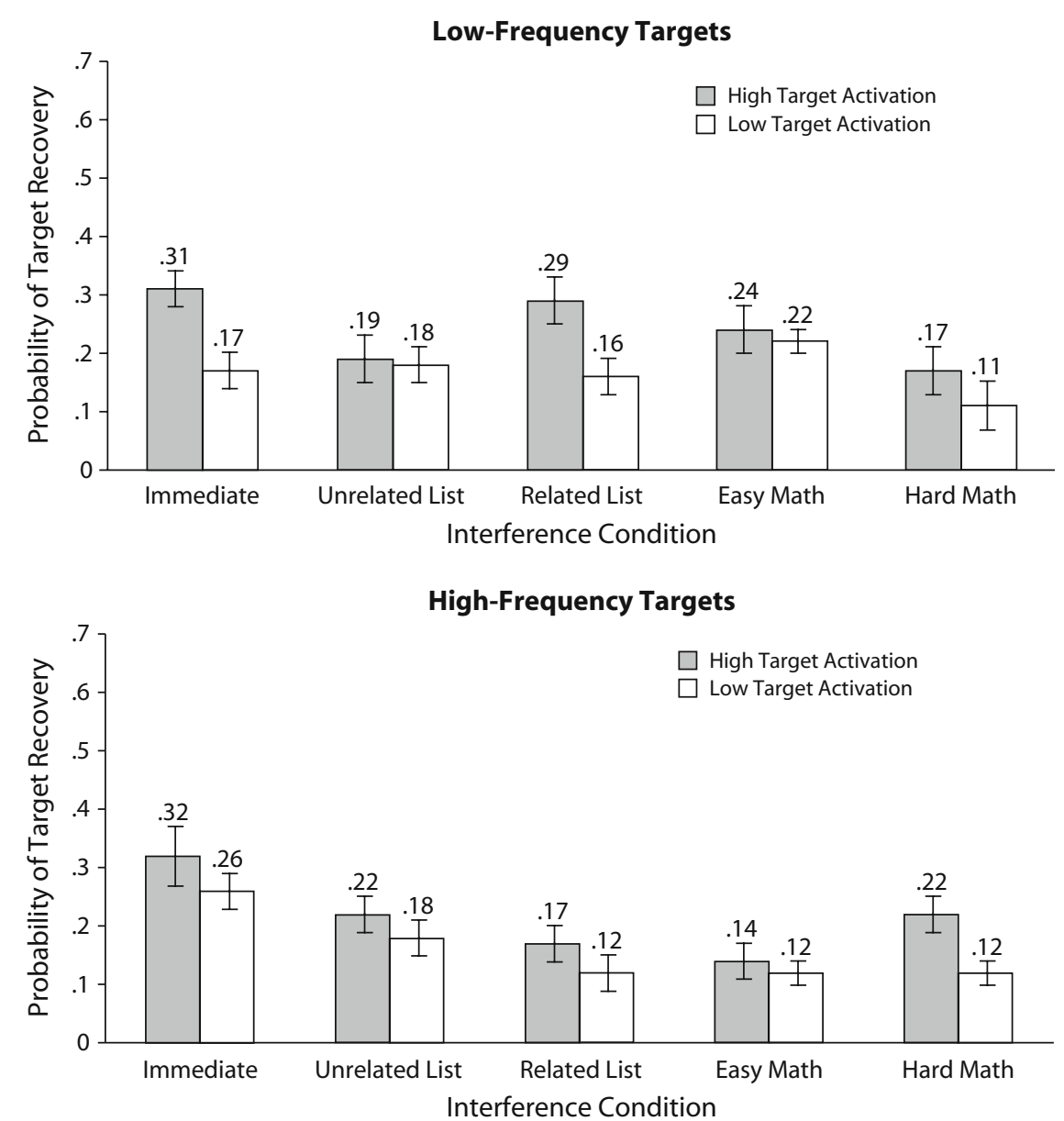

Figure 3. Probability of target recovery in primed free association in Experiment 1, as a function of context interference, target frequency, and target activation strength.

pattern was evident in Figure 3, but the probability of target recovery was much lower for primed free association than for cued recall. Although a recovery advantage for targets having stronger activation strengths was evident numerically in every condition, the advantage tended to be greater for immediate testing and cued recall.

A $5 \times 2 \times 2 \times 2$ mixed-model ANOVA of these data indicated that each variable had a significant effect on target recovery. The effect of interference indicated that recall was higher when testing was immediate (.38) than when it was delayed by easy math (.27), hard math (.27), studying a related list (.27), or studying an unrelated list (.30) $\left[F(4,300)=9.96, M S_{\mathrm{e}}=.03\right]$. The planned two-tailed Fisher's LSD of .04 indicated that recall was significantly higher on the immediate test than after each interference condition and that none of the differences between the interference conditions were reliable. The analysis also showed that the context awareness manipulation was significant $[F(1,300)=219.08]$. Probability of target recovery in cued recall (.40) was higher than in primed free association (.20). Furthermore, low-frequency targets (.32) were more likely to be recovered than were high-frequency targets $(.28)[F(1,300)=9.82]$, and importantly, high activation strength targets (.46) were significantly more likely to be recalled than were low activation strength targets $(.34)\left[F(1,300)=94.72, M S_{\mathrm{e}}=.014\right]$.

Only two interaction effects met the .05 criterion for significance in this analysis. The main result of Experiment 1 indicated that the effects of target activation strength were reduced by disruptions designed to reduce the effectiveness or the effective use of context cues during retrieval. The effects of target activation strength interacted with both interference condition $[F(4,300)=3.93]$ and context awareness $[F(1,300)=8.36]$. The interaction with interference condition arose because the effects of target activation strength were less apparent after delayed testing than after immediate testing. When testing immediately followed study, recall was considerably higher when target activation was strong (.46) than when it was weak (.30). When delayed, the relative advantage of stronger target activation diminished in all interference conditions. Recall probabilities for strongweak activation levels were .29-.25 for easy math and .30-.23 for hard math. Recall for strong-weak activation levels was .32-.22 for related lists and .34-.26 for unrelated lists. The LSD of .04 indicated that target activation level had a significant effect in each condition. The interaction with interference condition occurred because the effects of strong target activation were smaller when testing was 
delayed. How testing was delayed made little difference in this experiment. Contrasts between easy versus hard math, related versus unrelated lists, and math versus additional list learning revealed minor differences.

The significant interaction of target activation strength $\times$ context awareness was similar in form. Target activation strength had a stronger effect in extralist cued recall than in primed free association. In cued recall, the probabilities of target recovery for strong-weak levels of target activation strength were .46 and .34 , respectively, whereas in primed free association, they were .23 and .16 . As with interference condition, target activation strength has significant effects in each task (LSD $=.03$ ), but the magnitude of the effects was larger when the learning and testing conditions fostered the encoding and retrieval of context than when they did not.

None of the remaining interactions had significant effects, but two are of interest. First, unlike target activation strength, frequency failed to interact with the interference manipulation. Second, unlike target activation, frequency failed to interact with task, although there was a trend showing that low-frequency words were more likely to be recalled in cued recall than in primed free association. These null effects are merely suggestive, but they imply that the effects of printed frequency are more likely to be caused by distinctive letter features (Malmberg, Steyvers, Stephens, \& Shiffrin, 2002) than by priming (Nelson et al., 1998; Nelson $\&$ Goodmon, 2002). The mechanisms underlying target activation strength and frequency effects may be different.

The main findings of Experiment 1 indicate that the effects of target activation strength are reduced when the retrieval of context is disrupted by a test delay filled by problem solving or additional list study. Such effects are also reduced when the learning and testing task reduces the likelihood of encoding and retrieving context. These findings indicate that implicitly activated memories become associated to context. Other findings indicate that the type of interference can have no effect on either cued recall or primed free association. Problem solving difficulty has no measurable influence on target activation strength effects, presumably because the context for easy and difficult problems is similar. In the same vein, the relatedness of the words of the interference lists to the test cues can have no effect on target recovery and on the magnitude of target activation strength effects. In this experiment, such effects are reduced equally, regardless of whether the interference words are related to the test cues. Given that the test cues are as strongly related to the words in the related interference list as they are to the targets, the absence of a relatedness effect and an interaction with target activation strength appear to be at odds with the interactive cue assumption. If extralist and context cues jointly determine recall, interference effects and greater context mismatching should have been found for the related list. That they were not implies that either the theory is incorrect or the conditions were insufficient for generating the expected effects. For example, the targets of the interference list may have been too weakly encoded to produce the expected relatedness effects.
The results of Experiment 1 also indicate that 8 min of multiplication and $1.2 \mathrm{~min}$ of studying a second list can produce equivalent levels of target recall and equivalent effects on target activation strength. Spending more time on multiplication produces the same effects as spending less time on studying a second list. The context differentiation between multiplication problems and studying an interference list is most probably large, and this finding suggests that lengthening the retention interval compensates for reduced context mismatching in problem solving. The length of the retention interval and the nature of the interfering task appear to act together to determine context losses. In context-modified PIER2, tasks with different probabilities of context mismatching can be set to generate equivalent interference effects by changing the retention interval. However, implementing the trade-off in Experiment 1 forestalls conclusions about the separable effects of type of interference task and test delay. Experiment 2 was designed to address this problem, as well as the relatedness issue.

\section{EXPERIMENT 2}

Experiment 2 compares interference effects produced by using problem solving and list-learning tasks without confounding retention interval. Extralist cued recall is evaluated after short delays of $1.2 \mathrm{~min}$ and $4.0 \mathrm{~min}$ that are comparable to the short delays occurring when a single list is studied as an interference list. Context-modified PIER2 predicts target activation strength effects on immediate recall, and their decline with increases in the retention interval. Given that context mismatches are more likely after learning a second list than after multiplication, the model also predicts that the reduction of target activation effects will be more apparent after list learning than after problem solving. Studying a second list differentially decreases the probability of sampling the first list context, and this decrease is predicted to be particularly evident when the words of the interference list are related to the test cues. This experiment increased the chances of observing a relatedness effect by changing the way in which the delay was implemented. Rather than increasing the number of interference lists studied during the delay (Nelson, Bennett, et al., 1993), we created the longer delay by providing more study time on a single interference list. Longer study time on the related interference list should differentially increase the probability of interference effects and context mismatching in comparison with the unrelated list.

\section{Method}

Design and Participants. The main design formed a $3 \times 2 \times 2$ mixed-model factorial. We varied task (problem solving, unrelated lists, related lists) and retention interval (1.2 $\mathrm{min}, 4 \mathrm{~min}$ ) between participants, and target activation strength (strong, weak) within participants. We ran an immediate test control to determine the effects of problem solving at short retention intervals. Twenty-four participants served in each of the six between-subjects conditions, and another 24 served in the immediate testing control. The participants were assigned to conditions and rewarded for service as in the previous experiment. 
Materials and Procedure. We reused the low-frequency lists, easy math problems, and the extralist cuing procedures of Experiment 1 . At the 4-min delay, we equated retention interval in the listlearning and math tasks by increasing study time from the usual 3 -sec rate to $10 \mathrm{sec}$ for the unrelated and related interference lists and by providing more problems in the math task. Providing more study time on the interference list both increases the retention interval for the critical target list and simultaneously strengthens the encoding of words in the interference lists.

\section{Results and Discussion}

Problem solving at short retention intervals. Before proceeding to the main findings, we compared recall on the immediate test to recall after the math task, to determine the effects of problem solving at short retention intervals. Probability of recall on the immediate test was .64 and .47 , respectively, for strong and weak target activation strengths. In a $3 \times 2$ mixed-model analysis, data for the immediate test was analyzed with data for the short and long retention intervals of the math conditions in Figure 4. Target activation strength had significant effects $\left[F(1,69)=67.25, M S_{\mathrm{e}}=.015\right]$, indicating that recall was higher when activation was stronger (.61) than when it was weaker $(.44)$. Neither task $[F(2,69)=1.08]$ nor retention interval $[F(2,69)=1.61]$ had reliable effects.
These results indicated that reductions in target activation effects occurring after 8 min of math in Experiment 1 were not caused solely by context mismatching generated by having to switch processing from the study list to the math task and then back to the study list at test (Nelson, McEvoy, et al., 1993; Nelson et al., 1998). Such switching was also required at the shorter retention intervals in Experiment 2, but target activation strength effects were not significantly altered. The reductions in target activation strength effects observed at the 8-min interval in Experiment 1 but not at the shorter intervals of Experiment 2 depend more on the length of the retention interval than on context mismatching effects.

Main findings. Figure 4 indicates that target activation strength effects were apparent after all interference conditions. In contrast to the results of Experiment 1, they tended to be smaller after studying a related list than after studying an unrelated list or solving multiplication problems. The smallest effect of target activation occurred at the longer retention interval, which allowed $10 \mathrm{sec}$ of study for each interference word. The results of a $3 \times 2 \times$ 2 mixed-model ANOVA on these data indicated that recall was highest after problem solving (.51), next highest after studying an unrelated list (.45), and lowest after study-

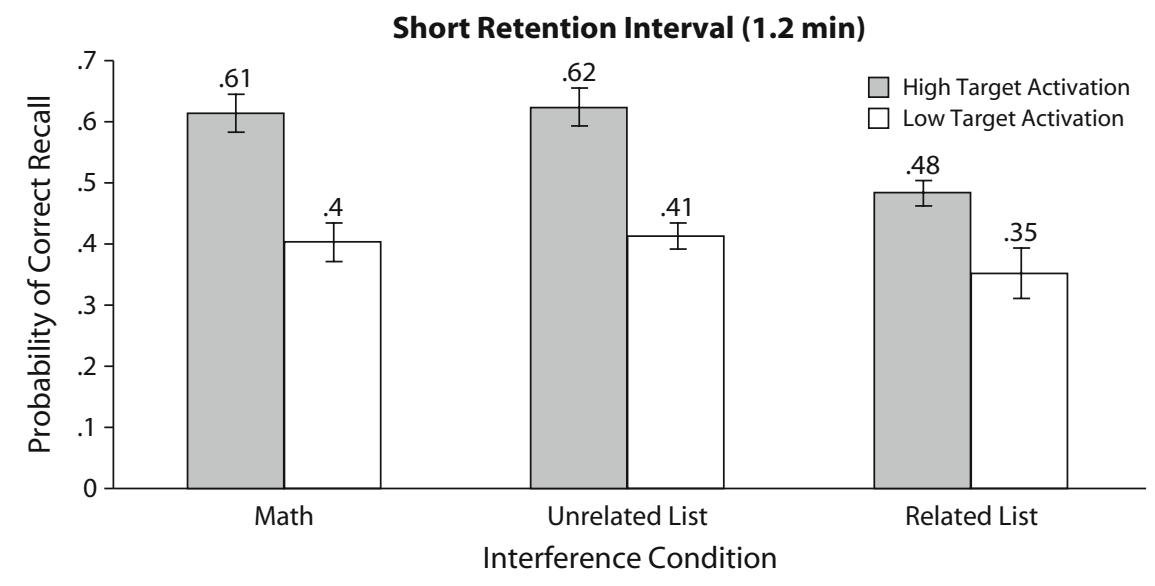

Long Retention Interval (4.0 min)

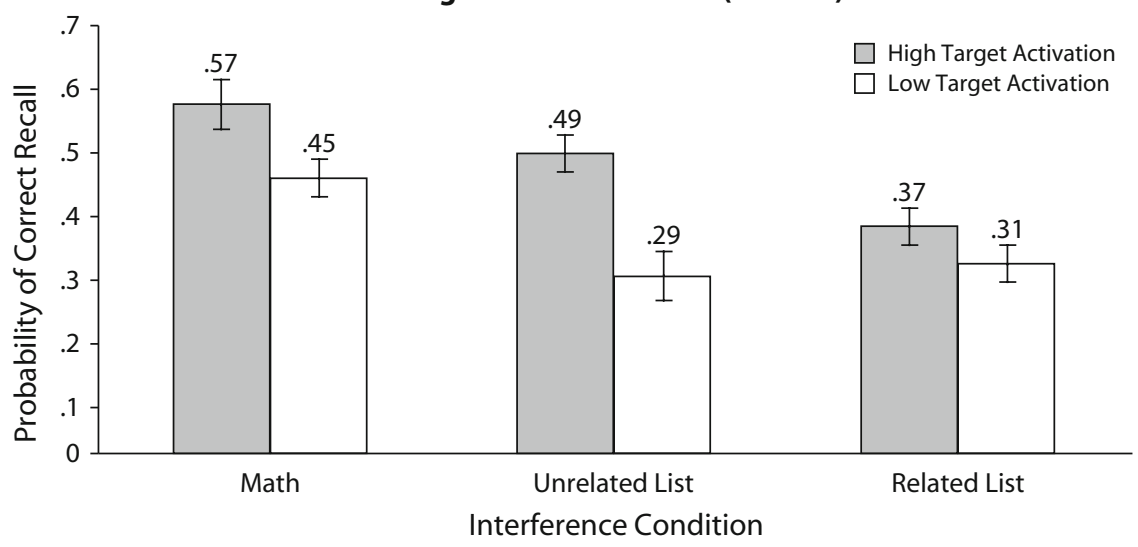

Figure 4. Probability of extralist cued recall in Experiment 2, as a function of context interference, retention interval, and target activation strength. 
ing a related list $(.38)\left[F(2,138)=12.38, M S_{\mathrm{e}}=.033\right]$. Each difference was significant $(\mathrm{LSD}=.05)$. When delay was controlled, studying an interpolated word list produced more interference than solving multiplication problems over comparable periods. In addition, recall was lower after studying a related list than after studying an unrelated list. Recall was significantly reduced when the words in the interference list were as strongly related to the test cues as were the target words.

This analysis also indicated that recall was higher when target activation was stronger (.52) than when it was weaker $(.37)\left[F(1,138)=111.22, M S_{\mathrm{e}}=.016\right]$. More importantly, the effects of target activation strength were significantly smaller for the related interference condition than for the unrelated list and the problem solving conditions $[F(1,138)=$ 5.42]. Recall for strong and weak levels of target activation for the related, unrelated, and math conditions are .42-.33, $.55-.35$, and .59-.42, respectively. Each difference is significant ( $\mathrm{LSD}=.05)$, but the magnitude of the difference is smaller after a related interference list has been learned. This finding is consistent with the context-modified model, because it implies that extralist and context cues jointly affect the probability of recovering the target. The threeway interaction among these variables was not significant $(F<1)$. Despite a trend in this direction, the target activation $\times$ relatedness interaction was not more apparent at the longer delay.

The statistical analysis of the findings shown in Figure 4 also indicates that recall was higher after the 1.2min retention interval (.48) than after the 4.0-min interval $(.42)[F(1,138)=8.99]$, but two interactions involving test delay were significant. First, the recall advantage for high in comparison with low target activation words found at the 1.2-min delay was reduced after a 4-min delay $[F(1,138)=3.75, \mathrm{LSD}=.04]$. Mean recall probabilities for strong and weak target activation words at the short delay were .57 and .39 , respectively. At the long delay, they were .48 and .35 . Second, delay effects were absent after the math task but were apparent after both list-learning tasks $[F(2,138)=3.50, \mathrm{LSD}=.07]$. The contrast means for the shorter and longer delays for related lists, unrelated lists, and math are .41-.34, .51-.39, and .50-.51, respectively.

Recall in this experiment varied as a function of both type of interference and test delay. When retention intervals are relatively short, additional list learning generates lower recall than does multiplication, and this result presumably occurs because list learning generates more context mismatches during retrieval than does problem solving. Similarly, learning a related list produces lower recall than learning an unrelated list because it generates more context mismatching. What is more important, the effects of implicitly activated memories on recall are moderated by both type of interference and length of the retention interval. The effects of implicitly activated memories on recalling the target that activated them decline with increases in the likelihood of context mismatching and test delay. Like memories created through elaborative processing, implicitly activated memories appear to be associated with the general contextual surround.

\section{GENERAL DISCUSSION}

The present findings add to a literature showing that a word's associative structure affects its recall when prompted by an extralist cue. When a word is encountered in isolation or in an ambiguous semantic context, the brain provides an internal context by activating the word's past interactions with other words in other contexts (Nelson \& McEvoy, 2007). Because of word learning experiences in thousands of contexts, words are both locally and globally entangled to varying degrees with associated words. They are locally entangled and produce locally organized associative sets because of direct links formed when the referents of the words appear contiguously; for example, radio-music, radio-stereo, music-stereo, stereo-music, and so forth. Locally entangled sets become globally entangled for the same reason; for example, music-art, art-paint, art-picture, paint-picture, and so forth. In the running example, radio and picture are not directly associated, but they are associated within a few links at a global level.

Entanglement at each level provides short paths or shortcuts to related words (Steyvers \& Tenenbaum, 2005), because words are organized as "small world" networks, much like computer (Barabási, 2002) and social (Strogatz, 2003; Watts \& Strogatz, 1998) networks. All isolated words provide implicit access to related associates that contextualize their meaning, and locally more entangled words recover more preexisting links or more associative context than do those that are less entangled. PIER2 assumes that a word's activation strength is determined by summing its entanglement history with closely related words and predicts that words with more and stronger preexisting associate-to-associate and associate-to-target links are more likely to be recalled in the presence of associatively related test cues.

Until recently (Nelson et al., 2007), PIER2 assumed that context is associated with explicit encoding operations applied to the target (e.g., rehearsal) but not to what it implicitly activates in long-term memory (Nelson et al., 1998). The present findings and other recent findings indicate that this assumption is incorrect. Recent findings show that the effects of increases in recall produced by stronger links of every type are reduced by disruptions that decrease the influence of context. The benefits of direct cue-to-target links (forward strength) and targetto-cue links (backward strength) decline as the retention interval increases from immediate testing to $20 \mathrm{~min}$ of multiplication to $20 \mathrm{~min}$ of multiplication plus $24 \mathrm{~h}$ of additional delay (Nelson \& Goodmon, 2002). The same article shows that the effects of indirect links that join the cue and target also decrease as the length of the retention interval increases. Related work indicates that the effects of target-to-cue as well as associate-to-cue links decline as retention interval increases, when social and environmental cues are changed from study to test, and when primed free association is used rather than the cued recall (Goodmon \& Nelson, 2004; Nelson et al., 2007). Recall benefits produced by direct and indirect links diminish (1) when context information becomes less acces- 
sible after a multiplication-filled delay, (2) after a context change from study to test, and (3) when the task reduces the likelihood of recovering context.

The present research shows that links that are directly connected to the target but not to the test cue are also affected by disrupting context. The results indicate that the effects of target activation strength determined mainly by the target's associate-to-associate links diminish after a short delay in the primed free association task. In this task, learning is incidental, the relation between the test cues and the words of the study context is never mentioned, and the short delay is produced only by test instructions and study-test lags. The reduction of target activation strength effects in this task suggests that using context cues during testing is not an automatic process. If it were automatic, probability of recall in primed free association should approach recall levels found in cued recall, but it did not. The likelihood of using context cues during testing varies with the intentions of the participants, and context cues are more likely to be used when the conditions identify a specific context than when they do not.

Target activation strength effects also diminish as a function of delay and how that delay is filled. As with links that join the test cue and target, the findings of Experiments 1 and 2 show that longer delays reduce target activation strength effects. The magnitude of the reduction, however, can depend on the interference tasks used to fill the delay. Both the retention interval and the task used to fill it affect recall. Solving multiplication problems reduces the effects of target activation strength after 8 min but not after 1.2-4 min of activity. In contrast, the results of Experiments 1 and 2 show that studying another list of words can reduce these effects after only $1.2 \mathrm{~min}$. At relatively short retention intervals, studying a second list of words can reduce the magnitude of target activation effects more than solving multiplication problems, but this reduction depends on the relatedness of the interference list words to the test cues.

In Experiment 2, the cued recall task focused participants on recalling words from the first list context. According to context-modified PIER2, recall is jointly determined by both context and extralist cues. The results show that the effects of target activation strength are differentially reduced for related in comparison with unrelated lists and multiplication. When the test cue is strongly associated to the target and to a word from the interference list, overall recall is less likely and the effects of target activation strength are differentially reduced. When the extralist cue directly recovers words appearing in two different list contexts, the likelihood of context mismatching is greater in comparison with unrelated lists and problem solving. In sharp contrast, for problem solving, the contexts produced by studying the target list and multiplying numbers are relatively distinct, and although context mismatches can occur, they are unlikely. For unrelated lists, context mismatching is more likely, because context switching occurs on very similar tasks. For unrelated lists, participants must switch processing from the second study list context to the first study list context during retrieval. Context switching is likely to generate mismatching occurring when the context features of the interference list are sampled instead of the context features of the first list (Mensink \& Raaijmakers, 1988). Mismatching is more likely after long delays, but the likelihood of mismatching is smaller when it is generated by context cues alone, as opposed to both context and extralist cues. According to the model, greater context mismatching in the related list occurs because processing must be switched from the second list context to the first list context during retrieval, and because the extralist cue recovers words from both contexts.

The results of the present experiments indicate that activating a word's associative history in the context of an experimental episode links the activation to that context. The target's implicitly activated memories of interactions with other words as well as the explicit processing operations applied to the target during study become connected to context. In terms of context-modified PIER2, the target's implicit and explicit representations are associated to the learning context. Recovering that context during testing facilitates the retrieval of both representations, and this recovery is affected by context awareness, test delay, and how that delay is filled. Context accessibility is determined by the length of the retention interval in minutes raised to a negative power of a parameter that varies with the nature of the context disruption, $t^{-c}$.

The parameter $c$ affects the accessibility of context cues encoded with the target during the study trial. $c$ is inversely related to context accessibility, so the larger $c$ is, the lower the probability of recovering context. $c$ has a larger value for primed free association than for cued recall, because context cues available during testing are less likely to be utilized in constraining target recovery. At a given retention interval, $c$ will have a larger value for studying a second word list than for solving multiplication problems, and, under some conditions, when the test cue is strongly related to both the target and a word from the interference list.

$c$ is also sensitive to combinations of context disruptions. Experiment 1 shows that context awareness and intervening activities increase $c$ and that these two forms of context disruption have additive effects on target recovery. Although context is never mentioned in the primed free association task, a test delay filled by an intervening event further reduces the retrieval of context. Implicit tests of memory are subject to the same sources of interference as are explicit tests (Lustig \& Hasher, 2001), and context accessibility affects performance on both types of tests (Schooler, Shiffrin, \& Raaijmakers, 2001). Hence, context accessibility declines with increasing delays, the rate of decline is affected by the nature of the activities during these delays, and multiple context disruptions can have cumulative effects on target recovery.

Context-modified PIER2 multiplies the predicted probabilities of recall of the implicit and explicit representations by the context accessibility computation, $t^{-c}$. This operation weights each representation by the effects of retention interval, task instructions, and type of intervening task. This adjustment allows the model to predict the effects of these variables on the effects of each representation. Because the test trials in the present experiments 


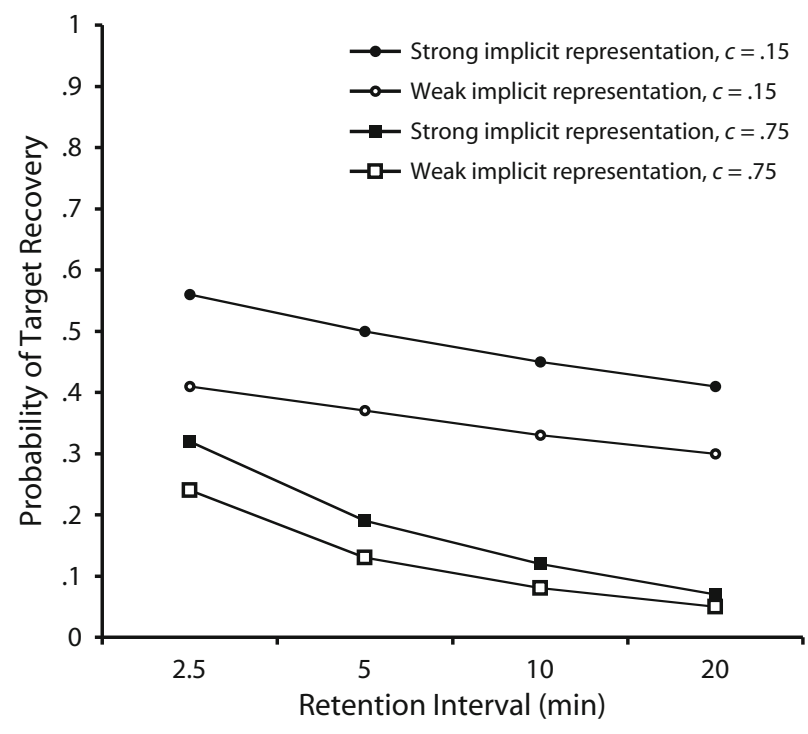

Figure 5. PIER2's predictions for target recovery, as a function of retention interval, context access parameter $c$, and the strength of the implicit representation.

were self-paced, we cannot make exact predictions; the exact retention interval varies somewhat across participants and is uncertain. However, the model's predictions for conditions that approximate those used in the present experiment are shown in Figure 5 to illustrate how the model explains the results. The effects of the explicit representation are ignored in order to concentrate on showing how the effects of the implicit representation of the target are altered by different tasks $(c)$ and by longer delays $(t)$. In PIER2, the probability of recovering the target's implicit representation is influenced by target activation strength, forward strength, and many other implicitly activated links (Nelson et al., 2007). Other than showing that higher levels of target activation strength increase the probability of recall, the details of this computation are unimportant here. What is important is that the probability of recovering the implicit representation of the target $(\mathrm{T})$ given extralist $(\mathrm{Q})$ and context $(\mathrm{C})$ cues is multiplied by context accessibility: $\mathrm{P}_{1}(\mathrm{~T} / \mathrm{Q}, \mathrm{C})\left(t^{-c}\right)$.

With this equation in mind, the probabilities of recovering the implicit representations for high and low levels of target activation strength on a zero delay test were set to .64 and .47 , respectively. Retention intervals were set from $2.5 \mathrm{~min}$ to $20 \mathrm{~min}$ by doubling the length of the delay at each step, and $c$ was set at .15 and .75 to mimic a wide range of context access losses, with high values of $c$ indicating high losses. The present experiments fall well within these conditions, and Figure 5 shows that the model predicts interactions between target activation strength and both retention interval and $c$. The recall advantage for higher target activation strength targets declines systematically as the length of the retention interval increases. Similarly, the recall advantage for higher activation strength targets will be less apparent when $c$ is high and mismatching is likely than when $c$ is low and mismatching is less likely. Furthermore, the model predicts that the effects of $c$ are relatively constant over the range of test delays displayed in Figure 5. For example, cued recall has a low relative value of $c$ in comparison with primed free association, so target recovery in cued recall is greater and is predicted to maintain this advantage within these delays.

The cumulative findings of this and other research indicate that a single word sometimes acts as if it were a collection of related words at an implicit, unconscious level of processing. The research also shows that this collective entity becomes associated to the context in which the processing occurs. Meaning accessible below the edges of consciousness interacts with the processing of the general information in its environment, perhaps equally below the edges of consciousness, and this interaction is encoded in ways that influence memory for a word that is seen and explicitly processed in elaborative ways. Context-modified PIER2 does reasonably well in predicting the effects that the collective entity has on recalling this word on an immediate test and after delays filled with various disruptive tasks and procedures. This success, however, is hollowed by the need to estimate $c$, a key parameter for determining context access after a disruption. Context access should be measurable, and although progress has been made (see, e.g., Mensink \& Raaijmakers, 1988), much remains to be done by way of definition, theory, and measurement.

\section{AUTHOR NOTE}

This research was supported by Grant MH16360 from the National Institute of Mental Health to D.L.N. We especially thank Cathy McEvoy and the reviewers for their constructive comments. Correspondence concerning this article should be addressed to D. L. Nelson, Department of Psychology, PCD 4118G, University of South Florida, Tampa, FL 33620-8200 (e-mail: dnelson@cas.usf.edu).

\section{REFERENCES}

Balota, D. A., Cortese, M. J., Sergent-Marshall, S. D., Spieler, D. H., \& YAP, M. J. (2004). Visual word recognition of single-syllable words. Journal of Experimental Psychology: General, 133, 283-316.

Barabási, A.-L. (2002). Linked: The new science of networks. Cambridge, MA: Perseus.

Gee, N. R., Nelson, D. L., \& KrawczyK, D. (1999). Is the concreteness effect a result of underlying network interconnectivity? Journal of Memory \& Language, 40, 479-497.

Goodmon, L. B., \& Nelson, D. L. (2004). Strengthening the activation of unconsciously activated memories. Memory \& Cognition, 32, 804-818.

Humphreys, M. S., Tehan, G., O’Shea, A., \& Bolland, S. W. (2000). Target similarity effects: Support for the parallel distributed processing assumptions. Memory \& Cognition, 28, 798-811.

Lustig, C., \& HASHER, L. (2001). Implicit memory is not immune to interference. Psychological Bulletin, 127, 629-650.

Malmberg, K. J., Steyvers, M., Stephens, J. D., \& Shiffrin, R. M. (2002). Feature frequency effects in recognition memory. Memory \& Cognition, 30, 607-613.

Mensink, G., \& RaAiJmakers, J. G. W. (1988). A model for interference and forgetting. Psychological Review, 95, 434-455.

Nelson, D. L., Bennett, D. J., Gee, N. R., Schreiber, T. A., \& MCKInNEY, M. (1993). Implicit memory: Effects of network size and interconnectivity on cued recall. Journal of Experimental Psychology: Learning, Memory, \& Cognition, 19, 747-764.

Nelson, D. L., \& Goodmon, L. B. (2002). Experiencing a word can prime its accessibility and its associative connections to related words. Memory \& Cognition, 30, 380-398.

Nelson, D. L., \& Goodmon, L. B. (2003). Disrupting attention: The need for retrieval cues in working memory theories. Memory \& Cognition, 31, 65-76.

Nelson, D. L., Goodmon, L. B., \& Ceo, D. (2007). How does delayed 
testing reduce effects of implicit memory: Context infusion or cuing with context? Memory \& Cognition, 35, 1014-1023.

Nelson, D. L., \& McEvoy, C. L. (2000). What is this thing called frequency? Memory \& Cognition, 28, 509-522.

Nelson, D. L., \& McEvoy, C. L. (2007). Entangled associative structures and context. In P. D. Bruza, W. Lawless, K. van Rijsbergen, \& D. A. Sofge (Eds.), Proceedings of the AAAI Spring Symposium on Quantum Interaction (pp. 98-105). Menlo Park, CA: AAAI Press.

Nelson, D. L., McEvoy, C. L., JanCZura, G. A., \& Xu, J. (1993). Implicit memory and inhibition. Journal of Memory \& Language, 32, 667-691.

Nelson, D. L., McEvoy, C. L., \& Pointer, L. (2003). Spreading activation or spooky action at a distance? Journal of Experimental Psychology: Learning, Memory, \& Cognition, 29, 42-52.

Nelson, D. L., McEvoy, C. L., \& Schreiber, T. A. (2004). The University of South Florida free association, rhyme, and word fragment norms. Behavior Research Methods, Instruments, \& Computers, 36, 402-407.

Nelson, D. L., McKinney, V. M., Gee, N. R., \& Janczura, G. A. (1998). Interpreting the influence of implicitly activated memories on recall and recognition. Psychological Review, 105, 299-324.

Nelson, D. L., \& Zhang, N. (2000). The ties that bind what is known to the recall of what is new. Psychonomic Bulletin \& Review, 7, 604-617.

Nelson, D. L., Zhang, N., \& McKinney, V. M. (2001). The ties that bind what is known to the recognition of what is new. Journal of Experimental Psychology: Learning, Memory, \& Cognition, 27, 1147-1159.
RaAijmakers, J. G. W., \& Shiffrin, R. M. (1981). Search of associative memory. Psychological Review, 88, 93-134.

Schooler, L. J., Shiffrin, R. M., \& RaAijmakers, J. G. W. (2001). A Bayesian model for implicit effects in perception. Psychological Review, 108, 257-272.

Smith, S. M., \& Vela, E. (2001). Environmental context-dependent memory: A review and meta-analysis. Psychonomic Bulletin \& Review, 8, 203-220.

Steyvers, M., \& Tenenbaum, J. B. (2005). The large-scale structure of semantic networks: Statistical analyses and a model of semantic growth. Cognitive Science, 29, 41-78.

Strogatz, S. H. (2003). Sync: The emerging science of spontaneous order. New York: Hyperion.

Watts, D. J., \& Strogatz, S. H. (1998). Collective dynamics of "smallworld" networks. Nature, 393, 440-442.

\section{NOTE}

1. PIER2's equation for relating the effects of the target's implicit (I), explicit (E), and context (C) representations on the probability of recalling target $(\mathrm{T})$ with an extralist cue $\left(\mathrm{Q}_{\mathrm{q}}\right)$ and context as test cues is $\operatorname{Pr}\left(\mathrm{T} \mid \mathrm{Q}_{t}, \mathrm{C}\right)=\operatorname{Pr}\left(\mathrm{T} \mid \mathrm{I}_{t}, \mathrm{E}_{t}\right) t^{-c}$. Specific definitions showing how target activation strength influences I, along with a numerical example, can be found in Nelson, Goodmon, and Ceo, 2007. 
APPENDIX

Materials Used in Experiments 1 and 2

Low-Frequency Lists A and B

List A

\begin{tabular}{llll}
\hline \multicolumn{1}{c}{ Test Cues } & $\begin{array}{c}\text { High-Connectivity } \\
\text { Targets }\end{array}$ & $\begin{array}{c}\text { Related } \\
\text { Interference List }\end{array}$ & $\begin{array}{c}\text { Unrelated } \\
\text { Interference List }\end{array}$ \\
\hline ATOM & PROTON & NEUTRON & BLIND \\
BRANCH & LEAF & LIMB & CHURCH \\
CLARINET & TRUMPET & INSTRUMENT & ACCOUNT \\
AROMA & SCENT & ODOR & BIG \\
DWARF & MIDGET & ELF & JUDGE \\
CRUMB & COOKIE & CAKE & EMOTION \\
ROBBERY & THEFT & BURGLAR & SOMETIMES \\
NET & VOLLEYBALL & FISH & VIOLET \\
THREAD & SEW & STRING & USUAL \\
SHOVEL & DIG & RAKE & GIRL \\
WHISKEY & RUM & BOURBON & NIGHT \\
SOAK & DRENCH & SPONGE & CHANGE \\
& LOW-COnnectivity & \multicolumn{1}{c}{ Related } & Unrelated \\
\multicolumn{1}{c}{ TeSt CueS } & Targets & Interference List & Interference List \\
\hline ANTIDOTE & POISON & CURE & STOOL \\
CEMENT & GLUE & CONCRETE & LIMIT \\
BEARD & SHAVE & HAIR & STOVE \\
CHARACTERISTIC & TRAIT & PERSONALITY & WONDER \\
EGGS & SCRAMBLE & BACON & WINTER \\
FIGHTER & BOXER & PILOT & RUN \\
JUMP & HOP & LEAP & DAY \\
MAZE & PUZZLE & MOUSE & STOMACH \\
OIL & GREASE & GAS & GROUP \\
PIGEON & DOVE & ROBIN & BEHIND \\
PUPPY & KITTEN & BABY & FREE \\
WISE & OWL & INTELLIGENT & CIRCLE \\
\hline
\end{tabular}

List B

\begin{tabular}{llll}
\hline \multicolumn{1}{c}{ Test Cues } & $\begin{array}{c}\text { High-Connectivity } \\
\text { Targets }\end{array}$ & $\begin{array}{c}\text { Related } \\
\text { Interference List }\end{array}$ & $\begin{array}{c}\text { Unrelated } \\
\text { Interference List }\end{array}$ \\
\hline ASPHALT & PAVEMENT & CONCRETE & CHURCH \\
MUSHROOM & FUNGUS & VEGETABLE & LOAN \\
BUMP & BRUISE & HIT & KID \\
GEM & RUBY & JEWEL & LEAP \\
GULP & SIP & SLURP & CHOICE \\
HURRICANE & TORNADO & TYPHOON & LEARN \\
MEDICATION & PRESCRIPTION & PILL & WAGE \\
SNORKEL & SCUBA & DIVE & SHOW \\
OFFEND & INSULT & DEFEND & MUSIC \\
STEAL & THIEF & ROB & BEHIND \\
TEAM & SOCCER & GROUP & COMMON \\
TRIANGLE & GEOMETRY & CIRCLE & NIGHT \\
& LOW-CONnectivity & \multicolumn{1}{c}{ Related } & Unrelated \\
\multicolumn{1}{c}{ TeSt CueS } & Targets & Interference List & Interference List \\
\hline BARREL & PICKLES & BUCKET & BLEACH \\
ANTEATER & NOSE & ANIMAL & EXPLODE \\
DICTIONARY & THESAURUS & ENCYCLOPEDIA & STOOL \\
APPLIANCE & TOASTER & STOVE & MIDDLE \\
BEARD & SHAVE & FACE & TOP \\
FINGER & TOE & FINGERNAIL & PUBLISHER \\
MOTORCYCLE & HELMET & BIKE & PERSONALITY \\
PUFF & DRAGON & POWDER & COURT \\
EXCUSE & PARDON & REASON & EVENING \\
LODGE & SKI & MOTEL & PILOT \\
SLENDER & SKINNY & SLIM & LIMIT \\
WALNUT & PECAN & NUT & ANNUAL \\
\hline
\end{tabular}


APPENDIX (Continued)

List A

\section{High-Frequency Lists A and B}

\begin{tabular}{llll}
\hline \multicolumn{1}{c}{ Test Cues } & $\begin{array}{c}\text { High-Connectivity } \\
\text { Targets }\end{array}$ & $\begin{array}{c}\text { Related } \\
\text { Interference List }\end{array}$ & $\begin{array}{c}\text { Unrelated } \\
\text { Interference List }\end{array}$ \\
\hline BIBLE & RELIGION & CHURCH & ODOR \\
DEAF & HEAR & BLIND & NEUTRON \\
EXPENSE & COST & ACCOUNT & LIMB \\
HUGE & LARGE & BIG & INSTRUMENT \\
JURY & TRIAL & JUDGE & GOLD \\
MOOD & FEELING & EMOTION & ELF \\
NEVER & AGAIN & SOMETIMES & FISH \\
PURPLE & COLOR & VIOLET & BURGLAR \\
REGULAR & NORMAL & USUAL & RAKE \\
SISTER & MOTHER & GIRL & SPONGE \\
STAR & SUN & NIGHT & STRING \\
VARY & DIFFERENT & CHANGE & BOURBON \\
& LOW-COnnectivity & Related & Unrelated \\
TeSt Cues & Targets & Interference List & Interference List \\
\hline BENCH & TABLE & STOOL & CURE \\
BOUNDARY & LINE & LIMIT & HAIR \\
CORRECT & RIGHT & WRONG & CONCRETE \\
CURIOSITY & INTEREST & WONDER & PUBLISHER \\
FALL & DOWN & WINTER & BACON \\
HIDE & FIND & RUN & PILOT \\
HOUR & TIME & DAY & LEAP \\
HUNGRY & FULL & STOMACH & MOUSE \\
INDIVIDUAL & SINGLE & GROUP & GAS \\
REAR & FRONT & BEHIND & ROBIN \\
WISE & OLD & INTELLIGENT & MATH \\
SLAVE & LABOR & FREE & MIDDLE \\
\hline IIS & & &
\end{tabular}

List B

\begin{tabular}{llll}
\hline \multicolumn{1}{c}{ Test Cues } & $\begin{array}{c}\text { High-Connectivity } \\
\text { Targets }\end{array}$ & $\begin{array}{c}\text { Related } \\
\text { Interference List }\end{array}$ & $\begin{array}{c}\text { Unrelated } \\
\text { Interference List }\end{array}$ \\
\hline BORROW & TAKE & LOAN & PATH \\
BRAT & CHILD & KID & HIT \\
BIBLE & RELIGION & CHURCH & RUN \\
CAB & CAR & DRIVER & SLURP \\
DECIDE & THINK & CHOICE & PILOT \\
EDUCATION & COLLEGE & LEARN & PILL \\
SALARY & PAY & WAGE & VEGETABLE \\
TELEVISION & RADIO & SHOW & STOMACH \\
TONE & VOICE & MUSIC & DIVE \\
TREASURE & ISLAND & GOLD & RAKE \\
WEATHER & TEMPERATURE & STORM & BLIND \\
UNUSUAL & NORMAL & COMMON & LIMB \\
& LOW-COnnectivity & Related & Unrelated \\
TeSt Cues & Targets & Interference List & Interference List \\
\hline BLONDE & HAIR & BLEACH & ANIMAL \\
BOMB & NUCLEAR & EXPLODE & STOVE \\
CALCULATOR & NUMBERS & MATH & BUCKET \\
CORE & CENTER & MIDDLE & CURE \\
DESK & TABLE & TOP & LIMIT \\
EDITOR & CHIEF & PUBLISHER & CONCRETE \\
INDIVIDUAL & SINGLE & GROUP & FINGERNAIL \\
JUSTICE & PEACE & COURT & MOTEL \\
MORNING & EARLY & EVENING & BACON \\
PEST & CONTROL & INSECT & POWDER \\
WORKER & LABOR & JOB & SLIM \\
YEAR & TIME & ANNUAL & NUT \\
\hline
\end{tabular}

(Manuscript received December 6, 2006; revision accepted for publication March 19, 2007.) 